\title{
FREQUENCY DOMAIN SIMULTANEOUS EQUATIONS METHOD FOR ACTIVE NOISE CONTROL SYSTEMS
}

\author{
Kensaku Fujii ${ }^{1}$, Mitsuji Muneyasu ${ }^{2}$, and Yusuke Fujita ${ }^{3}$ \\ ${ }^{1}$ Division of Computer Engineering, University of Hyogo \\ 2167 Shosha, Himeji, 671-2280 Japan \\ phone: +(81)-792-67-4992, fax: +(81)-792-67-4992, email: fujiken@eng.u-hyogo.ac.jp \\ ${ }^{2}$ Faculty of Engineering, Kansai University \\ 3-3-35 Yamate-cho, 564-8680, Suita, Japan \\ phone: +(81)-6-6368-0788, fax: +(81)-6-6388-8843, email: muneyasu@ ipcku.kansai-u.ac.jp \\ ${ }^{3}$ Catsystem Corporation \\ 3-147-27 Higashi-shimmmachi, 671-1121, Himeji, Japan \\ phone: +(81)-792-37-2545, fax: +(81)-792-37-2475, email: fujita@catsystem.co.jp
}

\begin{abstract}
This paper presents a new method applied to feedfoward type active noise control systems. This method, named frequency domain simultaneous equations method, is based on a different principle from the filtered-x algorithm requiring a filter modelled on a secondary path from a loudspeaker to an error microphone. Instead of the filter, this method uses an auxiliary filter identifying the overall path consisting of a primary path, a noise control filter and the secondary path. This paper first presents a computer simulation result demonstrating that the convergence speed of the proposed method is much higher than that of the filtered-x algorithm, and finally, by using an experimental system, verifies that the proposed method can automatically recover the noise reduction effect degraded by path changes.
\end{abstract}

\section{INTRODUCTION}

The filtered-x algorithm [1] is widely applied to the feedforward type of active noise control (ANC) system [2]. This algorithm, however, has a well-known drawback. Actually, the algorithm requires a filter, called secondary path filter, exactly modelled on the secondary path from a loudspeaker to an error microphone, whereas the secondary path in practical systems is continuously changing. This path change inevitably increases the modelling error, and at worst, the ANC system thereby falls into uncontrollable state[3].

In ANC systems using the filtered-x algorithm, repeatedly identifying the secondary path is required at intervals. The essence of the difficulty in the repeated identification is that the feedforward type system involves two unknown paths: the secondary path and a primary path from a noise detection microphone to an error microphone. Nevertheless, in the feedforward type system, available signals for identifying the two paths are only outputs of the two microphones, which can provide only one equation. To identify the two paths under active noise control, a device for yielding another independent equation is requisite [4]. As such a device, [5] presents a way of feeding an extra noise to the loudspeaker. In practical systems, avoiding the feeding is desirable.

A few method capable of automatically recovering the noise reduction effect without feeding the extra noise hence have proposed [6]-[8]. However, [6] and [7] neglect the feedback path from the loudspeaker to the noise detection microphone. In addition, the noise reduction speed of [6] and [7] is slower than that of the filtered-x algorithm, and the processing cost of [7] and [8] is high. On the other hand, [9] shows that the simultaneous equations method proposed in [8] can successfully work on condition that the feedback path causes no howling. This paper reduces the processing cost by applying a frequency domain technique to the simultaneous equations method.

The simultaneous equations method uses an auxiliary filter instead of the secondary path filter. By using the auxiliary filter, the method identifies the overall path from the noise detection microphone, through the primary path, the noise control filter and the secondary path, to the error microphone. As inferred from the configuration of the overall path, the auxiliary filter can provide two independent equations when the different coefficient vectors are given to the noise control filter. The coefficient vector of the optimum noise control filter minimizing the output of the error microphone is obtained by solving the equations.

In practical systems, the estimated coefficient vector of the noise control filter involves a few error. By using the error, the simultaneous equations method repeatedly updates the coefficient vector, and thereby automatically recovers the noise reduction effect degraded by path changes. This paper first presents a simulation result demonstrating that the proposed method gives much higher noise reduction speed than that of the filtered-x algorithm, and finally, by using an experimental system, verifies the performance of the proposed method.

\section{SIMULTANEOUS EQUATIONS METHOD}

Figure 1 shows the configuration of the feedforward type active noise control system using the simultaneous equations method [8], where the transfer functions designate the following signals, filters and paths,

$N(z)$ Primary noise,

$P(z) \quad$ Primary path from the noise detection microphone, Md, to the error microphone, Me,

$C(z)$ Secondary path from the loudspeaker, $\mathrm{Sp}$, to the error microphone, Me,

$B(z) \quad$ Feedback path from the loudspeaker, Sp, to the noise detection microphone, $\mathrm{Md}$,

$\hat{B}(z) \quad$ Feedback control filter,

$H(z) \quad$ Noise control filter,

$S(z) \quad$ Auxiliary filter, 


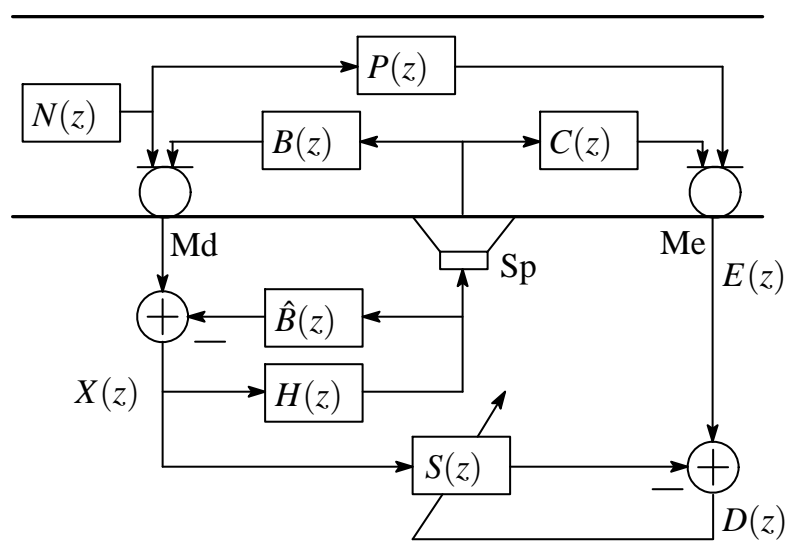

Figure 1 - Configuration of the feedforward type active noise control system using the simultaneous equations method

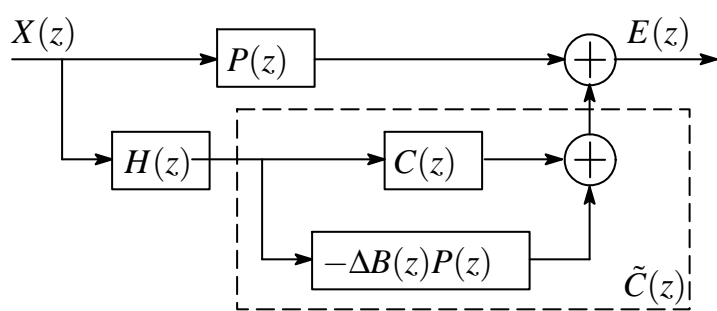

Figure 2 - Block diagram of the overall path identified by the auxiliary filter

$X(z) \quad$ Input signal of the noise control filter,

$E(z)$ Output signal of the error microphone,

$D(z) \quad$ Identification error,

respectively. The auxiliary filter $S(z)$ is used for identifying the overall path from the input of the noise control filter to the output of the error microphone, by using an adaptive algorithm. The overall path is moreover rearranged as shown in Fig. 2, where

$$
\Delta B(z)=B(z)-\hat{B}(z),
$$

and

$$
\tilde{C}(z)=C(z)-\Delta B(z) P(z) .
$$

Naturally, $\tilde{C}(z)$ is equal to $C(z)$ when the feedback control filter perfectly cancels the feedback path: $\Delta B(z)=0$. In any case, the auxiliary filter gives the following relation:

$$
S(z)=P(z)+H(z) \tilde{C}(z)
$$

as the result of the identification.

Here, our purpose is the derivation of the optimum noise control filter, $H_{\text {opt }}(z)$, satisfying the following relation:

$$
P(z)+H_{o p t}(z) \tilde{C}(z)=0 .
$$

Equation (2) states that the estimation of $P(z)$ and $\tilde{C}(z)$ is necessary for the derivation of $H_{o p t}(z)$. However, the available signals for the estimation are only $X(z)$ and $E(z)$, accordingly this system can provide only (1). As it stands, estimating $P(z)$ and $\tilde{C}(z)$ from only (1) is impossible. For the estimation, two independent equations are requisite.

To obtain the two equations, the simultaneous equations method exploits the fact that the system can give arbitrary coefficient vectors to the noise control filter if we accept the degradation of the noise reduction effect. The acceptance can provide us two relations,

$$
S_{1}(z)=P(z)+H_{1}(z) \tilde{C}(z)
$$

and

$$
S_{2}(z)=P(z)+H_{2}(z) \tilde{C}(z),
$$

after the identification of the overall path. Clearly, (3) and (4) are valid when $H_{2}(z) \neq H_{1}(z)$. On this condition, the elimination of $\tilde{C}(z)$ provides a solution,

$$
P(z)=\frac{S_{1}(z) H_{2}(z)-S_{2}(z) H_{1}(z)}{H_{2}(z)-H_{1}(z)},
$$

and similarly eliminating $P(z)$ from (3) and (4) gives another solution,

$$
\tilde{C}(z)=\frac{S_{1}(z)-S_{2}(z)}{H_{1}(z)-H_{2}(z)} .
$$

As mentioned above, our purpose is the estimation of $H_{\text {opt }}(z)$ satisfying (2). In addition, $P(z)$ and $C(z)$ necessary for calculating $H_{\text {opt }}(z)$ are obtained as (5) and (6). Then, the remained operation is only to substitute (5) and (6) into (2). The substitution yields the following equation:

$$
\begin{aligned}
& \left\{S_{1}(z) H_{2}(z)-S_{2}(z) H_{1}(z)\right\} \\
& -H_{o p t}(z)\left\{S_{2}(z)-S_{1}(z)\right\}=0 .
\end{aligned}
$$

Moreover, as inferred from Fig. 2, $H_{2}(z) \neq H_{1}(z)$ satisfies $S_{2}(z)-S_{1}(z) \neq 0$, therefore (7) gives

$$
H_{\text {opt }}(z)=\frac{S_{1}(z) H_{2}(z)-S_{2}(z) H_{1}(z)}{S_{2}(z)-S_{1}(z)}
$$

consisting of the known components.

\section{TRANSFORMATION INTO FILTER COEFFICIENT VECTOR}

In practical use, an operation for transforming $H_{\text {opt }}(z)$ into a filter coefficient vector is required. This paper applies a frequency domain technique to the transformation. The application of the fast Fourier transform (FFT) rewrites (8) as

$$
H_{o p t}(k)=\frac{S_{1}(k) H_{2}(k)-S_{2}(k) H_{1}(k)}{S_{2}(k)-S_{1}(k)}
$$

where $k$ is the element number of the frequency responses calculated by FFT.

On the other hand, (9) requires the estimation of the frequency response of the auxiliary filter, $S(k)$. For the estimation, this paper uses the following adaptive algorithm:

$$
S_{j+1}(k)=S_{j}(k)+\mu \frac{\sum_{i=j I+1}^{(j+1) I} D_{i}(k) X_{i}^{*}(k)}{\sum_{i=j I+1}^{(j+1) I} X_{i}(k) X_{i}^{*}(k)},
$$

where

j Block number,

$\mu \quad$ Step size,

$i \quad$ FFT duration number,

I The number of blocks,

$D_{i}(k) \quad k$ th spectrum element of the identification error $D(z)$ shown in Fig. 1, 


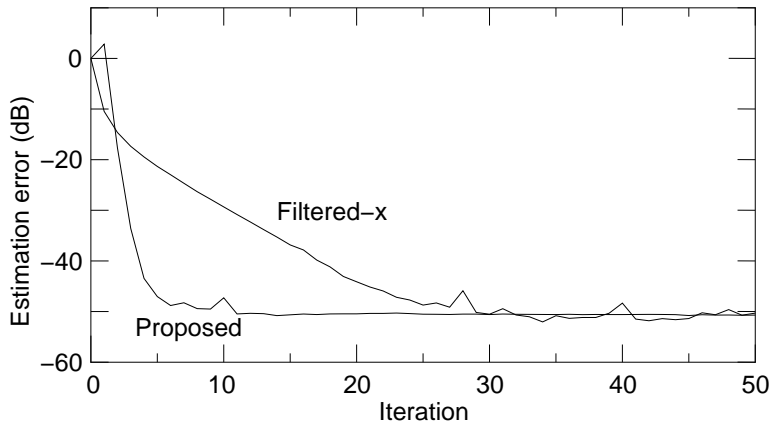

Figure 3 - Convergence properties provided by the filtered-x NLMS algorithm and the frequency domain simultaneous equations method

$X_{i}(k) \quad k$ th spectrum element of the noise control filter input $X(z), 0$

and $\{*\}$ designates the complex conjugate. The calculation of (10) is repeated $J$ times, and the results of the calculation are used as $S_{1}(k)$ and $S_{2}(k)$ in (9). In this algorithm, the block implementation is used for reducing the probability that the denominator of the second term becomes zero.

\section{UPDATING PROCEDURE}

In practical systems, giving arbitrary coefficient vectors to the noise control filter degrades the noise reduction effect. To prevent the degradation, the simultaneous equations method exploits the estimation error involved in the coefficient vector of the noise control filter. By using the estimation error, the simultaneous equations method can continuously refresh the coefficient vector. The automatic recovering of the degraded noise reduction effect thereby becomes possible.

The simultaneous equations method continuously updates the coefficient vector by repeating the procedures:

(1) Give a coefficient vector, $\boldsymbol{H}_{1}=\mathbf{0}$, to the noise control filter.

(2) Transform $\boldsymbol{H}_{1}$ into $H_{1}(k)$.

(3) Initialize the coefficient vector and frequency response of the auxiliary filter as $\boldsymbol{S}_{1}=\mathbf{0}$ and $S_{1}(k)=0$.

(4) Estimate $S_{1}(k)$ by using (10).

(5) Give another coefficient vector, for example,

$$
\boldsymbol{H}_{2}=\left[\begin{array}{llll}
1 & 0 & \cdots & 0
\end{array}\right]^{\mathrm{T}},
$$

to the noise control filter.

(6) Transform $\boldsymbol{H}_{2}$ into $H_{2}(k)$.

(7) Initialize the coefficient vector and frequency response of the auxiliary filter as $\boldsymbol{S}_{2}=\mathbf{0}$ and $S_{2}(k)=0$.

(8) Estimate $S_{2}(k)$ by using (10).

(9) Calculate $H_{\text {opt }}(k)$ by substituting the estimated $H_{1}(k)$, $H_{2}(k), S_{1}(k)$ and $S_{2}(k)$ into (9).

(10) Replace $S_{1}(k)$ with $S_{2}(k)$.

(11) Replace $H_{1}(k)$ with $H_{2}(k)$.

(12) Replace $H_{2}(k)$ with $H_{\text {opt }}(k)$.

(13) Transform $H_{o p t}(k)$ into $\boldsymbol{H}_{o p t}$.

(14) Give $\boldsymbol{H}_{\text {opt }}$ to the noise control filter as $\boldsymbol{H}_{2}$.

(15) Back to (6).

\section{COMPARISON WITH FILTERED-X NLMS ALGORITHM}

Here, this paper compares the convergence property of the proposed method with that of the filtered-x normalized least mean square (NLMS) algorithm. Fig. 3 is a simulation result calculated on the following conditions:

(1) The primary noise is generated by feeding white Gaussian noise to a filter whose transfer function is expressed as

$$
X(z)=1 /\left(1-2 \gamma \cos \theta z^{-1}+\gamma^{2} z^{-2}\right),
$$

where $\gamma=0.9$, and $\theta=\pi / 4$ corresponding to the resonance frequency of $1 \mathrm{kHz}$ when the sampling frequency is $8 \mathrm{kHz}$. Incidentally, this filter is modelled on the noise of the jet fan discharging exhaust gas to prevent it from filling in a tunnel.

(2) The feedback component is negligible: $\Delta B(z)=0$.

(3) The primary path is separable as $P(z)=A(z) C(z)$.

(4) Regular random numbers are given as the impulse response samples of the primary and secondary paths.

(5) The initial coefficient vectors of the noise control filter are $\boldsymbol{H}_{1}=\mathbf{0}$ and $\boldsymbol{H}_{2}=\left[\begin{array}{llll}1 & 0 & \cdots & 0\end{array}\right]^{\mathrm{T}}$, respectively.

(6) The number of taps of the noise control filter is 128.

(7) Impulse response sample numbers of the secondary and primary paths are 128 and 256, respectively. Accordingly, the duration of FFT is 512.

(8) $D_{i}(k)$ and $X_{i}(k)$ are calculated by using 256 samples of the identification errors

$$
\boldsymbol{D}_{i}=\left[0, \cdots, 0, d_{i}, \cdots, d_{i-255}\right]^{\mathrm{T}}
$$

and 512 samples of the noise control filter input signals

$$
\boldsymbol{X}_{i}=\left[x_{i}, \cdots, x_{i-255}, x_{i-256}, \cdots, x_{i-511}\right]^{\mathrm{T}} .
$$

(9) $S(k)$ estimated by using (10) is transformed into a coefficient vector

$$
\boldsymbol{S}=\left[s_{0}, \cdots, s_{255}, s_{256}, \cdots, s_{511}\right]^{\mathrm{T}},
$$

and then only the former 256 elements are given to the auxiliary filter.

(10) The later 384 elements of

$$
\boldsymbol{H}_{o p t}=\left[h_{0}, \cdots, h_{127}, h_{128}, \cdots, h_{511}\right]^{\mathrm{T}},
$$

estimated as the inverse FFT of (9) are discarded, and only the former 128 elements are given to the noise control filter.

(11) The step size applied to the filtered-x NLMS algorithm is 0.1 (the step size of more than 0.1 diverges the estimation error).

(12) $\mu=0.2, I=10$ and $J=10$ are applied to the proposed system; accordingly, the interval of updating the coefficient vector of the noise control filter is 51,200 sampling times. Here, it should be noted that these parameters are selected so that the estimation error converges on the same value that the filtered-x NLMS algorithm with the step size of 0.1 provides.

(13) The type of environmental noise is also white Gaussian.

(14) The power ratio of the primary noise to the environmental noise is $40 \mathrm{~dB}$. 


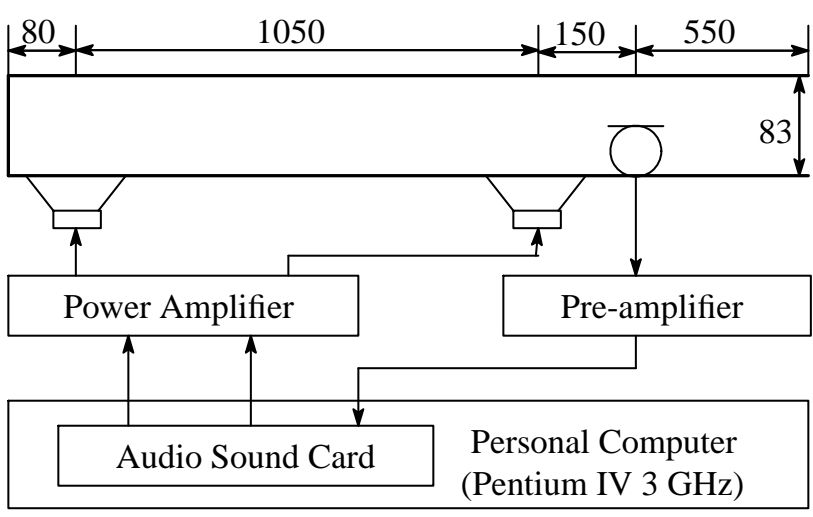

Figure 4 - Configuration of the experimental system used for verifying the performance of the proposed method

Table 1 - Equipments used in the experimental system

\begin{tabular}{|c|c|}
\hline Personal Computer & $\begin{array}{c}\text { Dell, Dimension 8300 } \\
\text { (Pentium IV 3GHz) }\end{array}$ \\
\hline Power Amplifier & Yamaha, HC-2700 \\
\hline Pre-amplifier & Audio Technica, AT-MA2 \\
\hline Loudspeaker & Pioneer, TS-E1076 \\
\hline Microphone & Audio Technica, AT-805F \\
\hline Audio Sound card & M Audio, Delta 44 \\
\hline
\end{tabular}

In addition, the horizontal axis in Fig. 3 shows the iteration number of updating the coefficient vector of the noise control filter. The updating interval is accordingly equivalent to $512 I J=51,200$ sample times. The vertical axis is also the estimation error involved in the coefficient vector of the noise control filter, which is calculated as

$$
\text { Error }=10 \log _{10}\left[\sum_{n=0}^{127}\left\{h_{n}-a_{n}\right\}^{2} / \sum_{n=0}^{127} a_{n}^{2}\right],
$$

where $h_{n}$ is the $n$th element of $\boldsymbol{H}_{\text {opt }}$ estimated by the proposed method, and similarly $a_{n}$ is the $n$th impulse response sample of the divided primary path $A(z)$.

This example shows that the convergence speed of the proposed method is much higher than that of the filtered-x NLMS algorithm. Here, it should be noted that the convergence property of the filtered-x NLMS algorithm is calculated on impractical assumption that the secondary path is perfectly identified with no error, and moreover the identification time of the secondary path is neglected.

\section{VERIFICATION BY EXPERIMENTAL SYSTEM}

By using an experimental system shown in Fig. 4, this paper finally verifies the performance of the proposed method. The experimental system is constructed with a vinyl chloride pipe of $83 \mathrm{~mm}$ diameter and controlled by a personal computer. Table 1 shows the main equipments used in the experimental system.

Figure 5 shows the decreasing properties of the error microphone output obtained by working the experimental system on the following conditions:

(1) The primary noise is a recorded diesel engine generator exhaust gas noise,

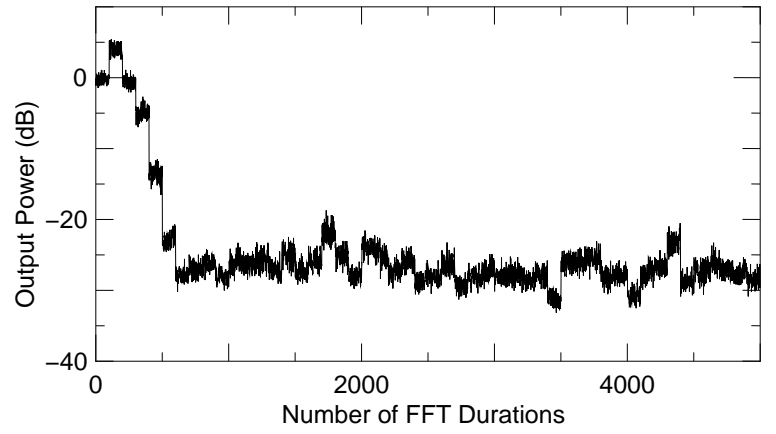

Figure 5 - Decreasing property of the error microphone output

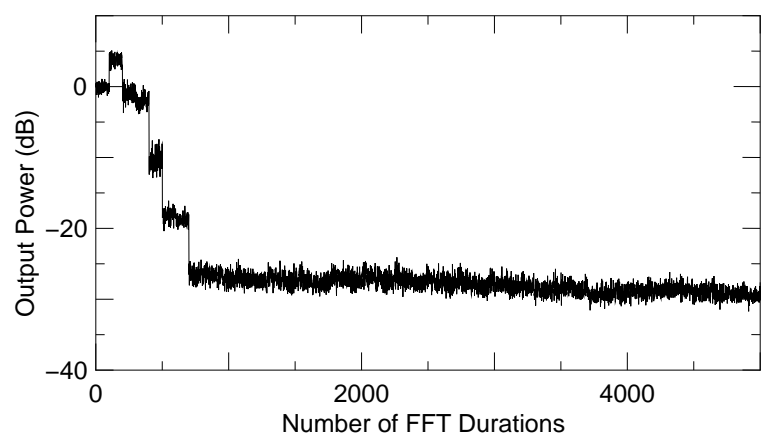

Figure 6 - Decreasing properties of the experimental system with the averaging operation

(2) The number of taps of the auxiliary filter is 1024 ,

(3) Accordingly, the duration of FFT is 2,048,

(4) The number of taps of the noise control filter is 512 ,

(5) $\mu=0.25, I=5, J=20$.

In Fig. 5, the horizontal axis designates the number of FFT durations, and the output power shown in the vertical axis is calculated as

$$
P e_{i}=10 \log _{10}\left[\sum_{k=0}^{1023} E_{i}(k) E_{i}^{*}(k) / P e_{0}\right],
$$

where $E_{i}(k)$ is the $k$ th spectrum component calculated by using the error microphone output samples in the $i$ th FFT duration, and $\mathrm{Pe}_{0}$ is calculated as

$$
P e_{0}=\sum_{i=0}^{I J-1} \sum_{k=0}^{1023} E_{i}(k) E_{i}^{*}(k) / I J,
$$

which is approximated to the average power of the error microphone output detected previous to feeding the secondary noise to the loudspeaker.

In this experiment, the first $200(=I J \times 2)$ FFT durations are used for only setting up the simultaneous equations, and the operation of updating the coefficient vector of the noise control is started from the 200th duration. According to the results, the output power of the error microphone decreases to less than $-20 \mathrm{~dB}$ after two or four updating operations, and this system stably keeps the output less than $20 \mathrm{~dB}$ after that.

On the other hand, the output power fluctuates in the durations after decreased to less than $-20 \mathrm{~dB}$, although inaudible. To reduce the fluctuation, this paper adds an operation 


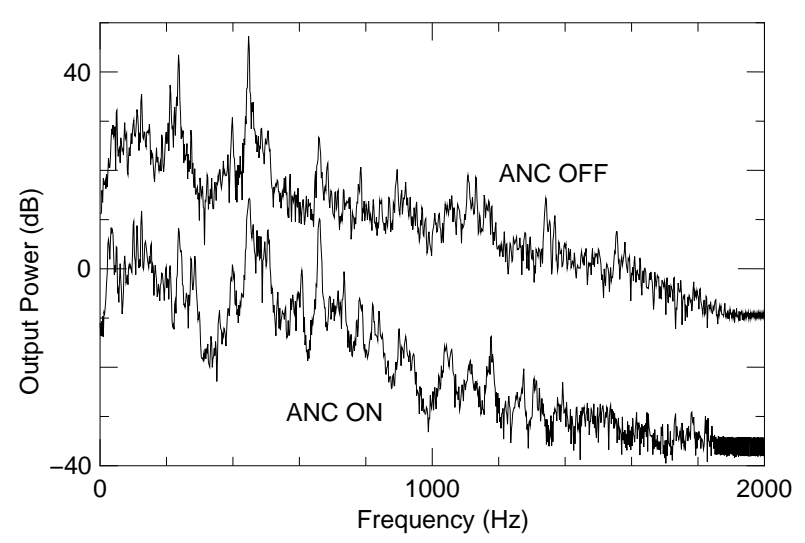

Figure 7 - Power spectrums of the error microphone outputs where "ANC ON" and "ANC OFF" denote after and before the application of the active noise control

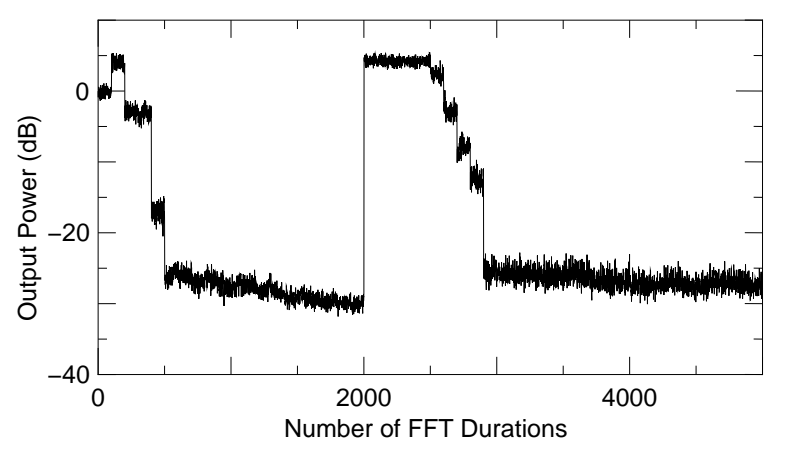

Figure 8 - Recovering property of the proposed method

of averaging the coefficient vector of the noise control filter to the proposed method. Fig. 6 is a decreasing property obtained by applying the averaging operation,

$$
\hat{H}_{\text {opt }}(k)=H_{\text {opt }}(k) \times 0.1+\hat{H}_{\text {opt }}(k) \times 0.9,
$$

to the proposed method only during the output power is less than $-20 \mathrm{~dB}$. Apparently, the averaging operation firmly keep the output power.

Next, let's confirm the frequency characteristics of the noise reduction effect provided by the proposed method. Figure 7 shows the average of the power spectrums calculated by using the error microphone output samples detected in five FFT durations, which is calculated as

$$
P a(k)=10 \log _{10}\left[\sum_{m=1}^{5} E_{m}(k) E_{m}^{*}(k) / I\right] .
$$

In this result, we can see that the noise reduction effect is obtained in all frequency range. Usually, the inversion of the effect is observed especially in the low and high frequency bands. In this experimental result, such inversion is not observed. This is an advantage of the proposed method.

The strong point of the proposed method is that the noise reduction effect degraded by path changes can automatically recover. This paper finally verifies the point by using the experimental system. Figure 8 shows the recovering property observed by using the experimental system, where the path change is substituted by multiplying the output of the noise control filter by -1 (change from $C(z)$ to $-C(z)$ ). This experimental result demonstrates that the proposed method successfully works in practical systems whose secondary path changes.

\section{CONCLUSION}

This paper has proposed the frequency domain simultaneous equations method capable of automatically recovering the noise reduction effect degraded by path changes. In addition, this paper has presented the simulation result demonstrating that the convergence speed of the method is much higher than that of the filtered-x NLMS algorithm and has verified the performance of the method by using the experimental system.

The simultaneous equations method can be also applied to updating the coefficient vector of the feedback control filter cancelling the feedback path from the secondary source to the noise detection microphone [9]. Our subsequent studies will hence focus on the application of the simultaneous equations method to the feedback type of active noise control system.

\section{REFERENCES}

[1] B. Widrow and S. D. Stearn, Adaptive signal processing. Englewood Cliffs, NJ: Printice Hall, 1985, pp. 288-294.

[2] S. J. Elliott and P. A. Nelson, "Active noise control," IEEE Signal Processing Magazine, vol. 10, no. 4, pp. 12-35, Sept. 1993.

[3] S. D. Synder and C. H. Hansen, "The effect of transfer function estimation errors on the filtered-x LMS algorithm," IEEE Trans. Signal Process., vol. SP-42, no. 4, pp. 950-953, April 1994.

[4] N. Saito and T. Sone, "Influence of modeling error on noise reduction performance of active noise control systems using filtered-x LMS algorithm," J. Acoust. Soc. Jpn (E), vol. 17, no. 4, pp. 195-202, July 1996.

[5] L. J. Erikson and M. C. Allie, "Use of random noise for online transducer modeling in an adaptive active attenuation system," J. Acoust. Soc. Am., vol. 85, no. 2, pp. 797-802, Feb. 1989.

[6] Y. Kajikawa and Y. Nomura, "An active noise control system without secondary path model," IEICE Trans. Fundamentals, vol. J82-A, no. 2, pp. 209-217, Feb. 1999.

[7] T. Khono, Y. Ohta and A. Sano, "Adaptive active noise control algorithm without explicit identification of secondary path dynamics," IEICE Trans. Fundamentals, vol. J86-A, no. 1, pp. 9-18, Jan. 2003.

[8] K. Fujii, M. Muneyasu and J. Ohga, "Active noise control systems by using the simultaneous equations method without estimation of the error path filter coefficients," IEICE Trans. Fundamentals, vol. J82-A, no. 3, pp. 299-305, March 1999.

[9] K. Fujii, Y. Iwamatsu, and M. Muneyasu, "A method to update the coefficients of feedback control filter," in Proc. ICSV12, Lisbon, Portugal, July 10-14, 2005, \#212. 